TRANSACTIONS OF THE

AMERICAN MATHEMATICAL SOCIETY

Volume 356, Number 10, Pages 4237-4254

S 0002-9947(04)03481-6

Article electronically published on May 10, 2004

\title{
ELLIPTIC APOSTOL SUMS AND THEIR RECIPROCITY LAWS
}

\author{
SHINJI FUKUHARA AND NORIKO YUI
}

\begin{abstract}
We introduce an elliptic analogue of the Apostol sums, which we call elliptic Apostol sums. These sums are defined by means of certain elliptic functions with a complex parameter $\tau$ having positive imaginary part. When $\tau \rightarrow i \infty$, these elliptic Apostol sums represent the well-known Apostol generalized Dedekind sums. Also these elliptic Apostol sums are modular forms in the variable $\tau$. We obtain a reciprocity law for these sums, which gives rise to new relations between certain modular forms (of one variable).
\end{abstract}

\section{INTRODUCTION}

Let $p$ and $q$ be relatively prime positive integers. First let us recall the definition of Apostol's generalized Dedekind sums $s_{k}(q, p)$ from [1], which we will call throughout this paper the Apostol sums: for a positive integer $k$,

$$
s_{k}(q, p):=\sum_{\mu=1}^{p-1} \frac{\mu}{p} \bar{B}_{k}\left(\frac{\mu q}{p}\right) .
$$

Here $\bar{B}_{k}(x)$ denotes the $k$-th Bernoulli function. That is, $\bar{B}_{k}(x)$ is given by the Fourier expansion

$$
\bar{B}_{k}(x):=-k ! \sum_{\substack{m=-\infty \\ m \neq 0}}^{+\infty} \frac{e^{2 \pi i m x}}{(2 \pi i m)^{k}} .
$$

It is well known that for $0 \leq x<1, \bar{B}_{k}(x)$ reduces to the $k$-th Bernoulli polynomial. We denote by $B_{k}$ the $k$-th Bernoulli number.

If $k$ is even, $s_{k}(q, p)$ is trivial. If $k$ is odd, a reciprocity law for the Apostol sums was obtained by Apostol [1 p. 149]:

$$
\begin{aligned}
p^{2 n-2} & s_{2 n-1}(q, p)+q^{2 n-2} s_{2 n-1}(p, q) \\
\quad= & \frac{1}{p q}\left\{\sum_{j=0}^{n} \frac{(2 n-1) ! B_{2 j} B_{2 n-2 j}}{(2 j) !(2 n-2 j) !} p^{2 j} q^{2 n-2 j}+\frac{(2 n-1) B_{2 n}}{2 n}\right\} \quad(n>1) .
\end{aligned}
$$

Received by the editors September 30, 2002 and, in revised form, August 7, 2003.

2000 Mathematics Subject Classification. Primary 11F20; Secondary 33E05, 11F11.

Key words and phrases. Generalized Dedekind sums (Apostol sums), elliptic functions, elliptic Apostol sums, modular forms, reciprocity laws.

The first author was partially supported by Grant-in-Aid for Scientific Research (C)12640089, Ministry of Education, Sciences, Sports and Culture, Japan.

The second author was partially supported by a Research Grant from NSERC, Canada. 
There are a number of different proofs for the Apostol's reciprocity law (1.1) (cf. [1, 2, 6, 16]). However, we believe that our approach [12] that makes use of the following trigonometric identity (1.2) provides an elegant proof.

For relatively prime positive integers $p$ and $q$, and for $z \in \mathbb{C}$, we have ( $\mathbf{7}$, Theorem 2.4], [11, 12])

$$
\begin{array}{r}
\frac{1}{p} \sum_{\mu=1}^{p-1} \cot \left(\frac{\mu q \pi}{p}\right) \cot \left(z+\frac{\mu \pi}{p}\right)+\frac{1}{q} \sum_{\mu=1}^{q-1} \cot \left(\frac{\mu p \pi}{q}\right) \cot \left(z+\frac{\mu \pi}{q}\right) \\
=-\cot (p z) \cot (q z)+\frac{1}{p q} \csc ^{2}(z)-1 .
\end{array}
$$

We are grateful to the referee for pointing out that this trigonometric sum is nothing but a special case of a reciprocity formula for the so-called DedekindRademacher sums (which are Dedekind sums with two congruence parameters $(z$ and 0 in (1.2))). Introduction of these congruence parameters into the classical Dedekind sums may be viewed as unifying all Apostol sums into a single sum. In fact, we expand both sides of (1.2) into Laurent series in $z$ and in addition we apply finite Fourier transforms to them. Then comparing the coefficients of $z^{2 n-2}$ of both sides, we arrive at the reciprocity law for the Apostol sums (1.1) in the case $n>1$. For $n=1$, this formula yields the reciprocity law for the classical Dedekind sums [18, p. 18]:

$$
\frac{1}{p} \sum_{\mu=1}^{p-1} \cot \left(\frac{\mu q \pi}{p}\right) \cot \left(\frac{\mu \pi}{p}\right)+\frac{1}{q} \sum_{\mu=1}^{q-1} \cot \left(\frac{\mu p \pi}{q}\right) \cot \left(\frac{\mu \pi}{q}\right)=\frac{p^{2}+q^{2}+1-3 p q}{3 p q}
$$

In view of the fact that (1.2) gives rise to the Apostol reciprocity law (1.1), we may regard the identity (1.2) as a "generating function" for the reciprocity law (1.1).

In this paper, we will define an elliptic analogue of the Apostol sums (Definition 2.1), using Weierstrass $\wp$ and $\zeta$-functions. It is defined as a finite sum of terms where every term is the product of two functions related to elliptic functions (Weierstrass $\wp$ and $\zeta$-functions). Its very definition depends in an essential way on the parity of two relatively prime positive integers $p$ and $q$ (see Remark 2.1. Definition 2.1 and Remark 2.2). Then we will investigate properties of these sums. First we obtain an elliptic analogue of the identity (1.2) (Theorem 2.1). (This new identity will serve as a "generating function" for a reciprocity law.) Then we establish a reciprocity law for these elliptic analogues (Theorem 2.2).

In the mid 80's, elliptic analogues of the classical Dedekind sums (so-called elliptic Dedekind sums) were studied by several authors (see [19, 14]). Earlier, higherdimensional (or multiple) Dedekind sums were discussed in the paper of Zagier [20]. As an elliptic analogue of Zagier's multiple Dedekind sums, higher-dimensional (or multiple) elliptic Dedekind sums were introduced and investigated, rather recently, by Egami [8], and also by Bayad [3. Unfortunately the reciprocity laws obtained by Egami ([즈, Theorem 1]) and Bayad ([3, Theorem 2.2]) for elliptic Dedekind sums were incorrect, i.e., the right-hand sides of their formulae should be divided by $a_{0} \cdots a_{n}$. (In the proof of Lemma 3.1 below (see also Remark 3.1), the reader will find a reason why their errors occurred.)

Under this circumstance, it would be desirable to establish our results (Theorem 2.1 and Theorem 2.2) independently from the papers of Egami and Bayad, and indeed, this is one of our purposes of the present paper. The strategy of our proof 
for Theorem 2.1 is to apply Liouville's theorem (that any bounded entire function on $\mathbb{C}$ must be constant), and it differs from the method employed by Egami [8] and Bayad [3] (who used the residue theorem).

We also calculate the limit of the elliptic reciprocity laws (2.3) and (2.4) at $i \infty$. We show that the limit formula indeed coincides with the reciprocity law (1.2) for Apostol sums. (This fact may justify our terminology "elliptic Apostol sums".)

Finally we will briefly interpret elliptic Apostol sums in terms of modular forms, and observe that they give rise to some new relations between modular forms. (We refer the reader who are interested in modular forms aspects of elliptic Dedekind sums to the paper of Bayad [3, where the author has carried out investigations on elliptic Dedekind sums and their reciprocity laws in terms of Jacobi forms.)

We should mention that the elliptic analogues of the Apostol sums treated in this paper are strictly speaking analogues and generalizations of the cotangent sums (1.2), which involve trigonometric sums (i.e., degenerate elliptic functions). It is still an open problem to find true analogues in the elliptic world of the classical Apostol sums which involve Bernoulli polynomials.

\section{THE MAIN RESUlts}

First we recall the three elliptic functions which play central roles in our discussion. Fix a complex number $\tau$ with positive imaginary part. The Weierstrass $\wp$-function (introduced by Weierstrass) is a meromorphic function of a complex variable $z$ given as follows:

$$
\wp(\tau, z):=\frac{1}{z^{2}}+\sum_{\substack{\gamma \in 2 \pi i(\mathbb{Z} \tau+\mathbb{Z}) \\ \gamma \neq 0}}\left(\frac{1}{(z-\gamma)^{2}}-\frac{1}{\gamma^{2}}\right) .
$$

Associated to $\wp(\tau, z)$, the function $e_{1}(\tau)$ is defined by putting $z=\pi i$ in $\wp(\tau, z)$ :

$$
e_{1}(\tau):=\wp(\tau, \pi i)
$$

It is known that the $\wp$-function has the following expansion at $z=0$ :

$$
\wp(\tau, z)=\frac{1}{z^{2}}+\sum_{n=2}^{\infty}(2 n-1) G_{2 n}(\tau) z^{2 n-2},
$$

where $G_{2 n}(\tau)$ is the Eisenstein series of weight $2 n$, namely,

$$
G_{2 n}(\tau):=\sum_{\substack{\gamma \in 2 \pi i(\mathbb{Z} \tau+\mathbb{Z}) \\ \gamma \neq 0}} \frac{1}{\gamma^{2 n}}
$$

Let $\Gamma(n)$ and $\Gamma_{0}(n)$ be the congruence subgroups of $S L_{2}(\mathbb{Z})$ defined respectively by

$$
\begin{aligned}
\Gamma(n) & :=\left\{\left(\begin{array}{ll}
a & b \\
c & d
\end{array}\right) \in S L_{2}(\mathbb{Z}) \mid a \equiv d \equiv 1(n), b \equiv c \equiv 0(n)\right\}, \\
\Gamma_{0}(n) & :=\left\{\left(\begin{array}{ll}
a & b \\
c & d
\end{array}\right) \in S L_{2}(\mathbb{Z}) \mid c \equiv 0(n)\right\} .
\end{aligned}
$$

It is well known that $G_{2 n}(\tau)(n>1)$ and $e_{1}(\tau)$ are modular forms for $S L_{2}(\mathbb{Z})$ and $\Gamma_{0}(2)$ of weight $2 n$ and 2 , respectively. 
The Weierstrass $\zeta$-function (also introduced by Weierstrass) is a meromorphic function of a complex variable $z$ given as follows:

$$
\zeta(\tau, z):=\frac{1}{z}+\sum_{\substack{\gamma \in 2 \pi i(\mathbb{Z} \tau+\mathbb{Z}) \\ \gamma \neq 0}}\left(\frac{1}{z-\gamma}+\frac{1}{\gamma}+\frac{z}{\gamma^{2}}\right) .
$$

Then it is easy to see that the two functions $\wp(\tau, z)$ and $\zeta(\tau, z)$ have the following relation:

$$
\partial \zeta(\tau, z) / \partial z=-\wp(\tau, z) .
$$

We see that the $\wp$-function $\wp(\tau, z)$ is periodic with respect to $2 \pi i(\mathbb{Z} \tau+\mathbb{Z})$. The $\zeta$-function $\zeta(\tau, z)$ is subject to the following identities:

$$
\zeta(\tau, z+2 \pi i)=\zeta(\tau, z)+\eta_{1}(\tau), \quad \zeta(\tau, z+2 \pi i \tau)=\zeta(\tau, z)+\eta_{2}(\tau),
$$

where $\eta_{1}(\tau)$ and $\eta_{2}(\tau)$ are independent of $z$. This implies that the function $\zeta(\tau, z)$ is not periodic but quasi-periodic with respect to $2 \pi i(\mathbb{Z} \tau+\mathbb{Z})$.

Now we define the function $\varphi(\tau, z)$ in a complex variable $z$ as follows:

$$
\begin{aligned}
& \varphi(\tau, z)=\sqrt{\wp(\tau, z)-e_{1}(\tau)}, \\
& \varphi(\tau, z)=\frac{1}{z}+O(1) \quad(z \rightarrow 0) .
\end{aligned}
$$

It is therefore easy to see that the function $\varphi(\tau, z)$ is periodic with respect to $2 \pi i(2 \mathbb{Z} \tau+\mathbb{Z})$ and that it has the following Laurent expansion at $z=0$ :

$$
\varphi(\tau, z)=\frac{1}{z} \sum_{n=0}^{\infty} H_{2 n}(\tau) z^{2 n},
$$

where $H_{2 n}(\tau)$ is determined recursively using (2.1):

$$
\begin{gathered}
H_{0}(\tau)=1, \quad H_{2}(\tau)=-\frac{1}{2} e_{1}(\tau) \\
H_{2 n}(\tau)=-\frac{1}{2} \sum_{j=1}^{n-1} H_{2 j}(\tau) H_{2 n-2 j}(\tau)+\frac{2 n-1}{2} G_{2 n}(\tau) \quad(n>1) .
\end{gathered}
$$

Clearly $H_{2 n}(\tau)$ is a polynomial in $e_{1}(\tau), G_{4}(\tau), G_{6}(\tau), \ldots, G_{2 n}(\tau)$ with rational coefficients. Furthermore, we see that $H_{2 n}(\tau)$ is a modular form of weight $2 n$ for $\Gamma_{0}(2)$. Also $\varphi(\tau, z)$ satisfies the following transformation formulae:

$$
\varphi(\tau,-z)=-\varphi(\tau, z)
$$

and

$$
\varphi(\tau, z+2 \pi i \tau)=-\varphi(\tau, z) .
$$

For a fixed $\tau$ with positive imaginary part, to ease the notations, we will simply write $\wp(z), \zeta(z), \varphi(z), e_{1}, \eta_{1}, \eta_{2}, G_{2 n}$ and $H_{2 n}$ dropping the variable $\tau$. We denote by $f^{\prime}(z)$ the first derivative of a function $f(z)$ with respect to $z$, and more generally, by $f^{(k)}(z)$ the $k$-th derivative of a function $f(z)$. 
Retaining these notations we can now formulate our results:

Theorem 2.1. Let $p$ and $q$ be relatively prime positive integers, and $z \in \mathbb{C}$.

(1) Suppose that $p+q \equiv 1$ (2). Then we have

$$
\begin{gathered}
\frac{1}{p} \sum_{\substack{\lambda, \mu=0 \\
(\lambda, \mu) \neq(0,0)}}^{p-1}(-1)^{\lambda} \varphi\left(\frac{2 \pi i q(\lambda \tau+\mu)}{p}\right) \varphi\left(z+\frac{2 \pi i(\lambda \tau+\mu)}{p}\right) \\
+\frac{1}{q} \sum_{\substack{\lambda, \mu=0 \\
(\lambda, \mu) \neq(0,0)}}^{q-1}(-1)^{\lambda} \varphi\left(\frac{2 \pi i p(\lambda \tau+\mu)}{q}\right) \varphi\left(z+\frac{2 \pi i(\lambda \tau+\mu)}{q}\right) \\
=-\varphi(p z) \varphi(q z)-\frac{1}{p q} \varphi^{\prime}(z) .
\end{gathered}
$$

(2) Suppose that $p+q \equiv 0$ (2). Then we have

$$
\begin{gathered}
\frac{1}{p} \sum_{\substack{\lambda, \mu=0 \\
(\lambda, \mu) \neq(0,0)}}^{p-1}(-1)^{\lambda} \varphi\left(\frac{2 \pi i q(\lambda \tau+\mu)}{p}\right) \zeta\left(z+\frac{2 \pi i(\lambda \tau+\mu)}{p}\right) \\
+\frac{1}{q} \sum_{\substack{\lambda, \mu=0 \\
(\lambda, \mu) \neq(0,0)}}^{q-1}(-1)^{\lambda} \varphi\left(\frac{2 \pi i p(\lambda \tau+\mu)}{q}\right) \zeta\left(z+\frac{2 \pi i(\lambda \tau+\mu)}{q}\right) \\
=-\varphi(p z) \varphi(q z)-\frac{1}{p q} \zeta^{\prime}(z)+C(p, q ; \tau)
\end{gathered}
$$

where $C(p, q ; \tau)$ is a constant with respect to $z$.

Remark 2.1. In Theorem 2.1, the parity conditions on the relatively prime positive integers $p$ and $q$ turn out to be rather essential. As we see, the only difference in two identities lies in the second factor on the right-hand side. Indeed, in (2.3), the second factor is the derivative of the $\varphi$-function, while in (2.4), the second factor is the derivative of the $\zeta$-function. If we drop the parity conditions, the identity (2.3) (resp. (2.4)) no longer holds true.

In fact, if the opposite parity for $p+q$ is taken in (2.3) (resp. (2.4)), the lefthand side and the right-hand side of the equation end up having different periods, namely, $2 \pi i(\mathbb{Z} \tau+\mathbb{Z})$ and $2 \pi i(2 \mathbb{Z} \tau+\mathbb{Z})$, respectively. This implies that the identity does not hold under this parity. For instance, (2.4) is not valid if we take $p=2$ and $q=1$, while (2.3) does not hold for $p=q=1$. Therefore, the parity condition on $p+q$ is rather essential for the validity of the identity.

Next we define the "elliptic Apostol sums" which we may regard as elliptic analogues of the Apostol sums.

We need two different types of elliptic Apostol sums depending on the parity conditions of relatively prime positive integers $p$ and $q$.

Definition 2.1. Let $p$ and $q$ be relatively prime positive integers, and let $k$ be a positive integer. 
Depending on the parity of $p+q$, we define the sum $\tilde{s}_{k}(q, p ; \tau)$ as follows:

$$
\begin{aligned}
& \tilde{s}_{k}(q, p ; \tau) \\
& ::=\left\{\begin{array}{l}
-\frac{k}{p^{k}} \sum_{\substack{\lambda-1 \\
\lambda, \mu=0 \\
(\lambda, \mu) \neq(0,0)}}^{\substack{k \\
p^{k}}}(-1)^{\lambda} \varphi\left(\frac{2 \pi i q(\lambda \tau+\mu)}{p}\right) \varphi^{(k-1)}\left(\frac{2 \pi i(\lambda \tau+\mu)}{p}\right)(\text { if } p+q \equiv 1(2)), \\
(\lambda, \mu) \neq(0,0)
\end{array}(-1)^{\lambda} \varphi\left(\frac{2 \pi i q(\lambda \tau+\mu)}{p}\right) \zeta^{(k-1)}\left(\frac{2 \pi i(\lambda \tau+\mu)}{p}\right)(\text { if } p+q \equiv 0(2)) .\right.
\end{aligned}
$$

We call $\tilde{s}_{k}(q, p ; \tau)$ the elliptic Apostol sum.

Remark 2.2. We adopt the notation $\tilde{s}_{k}(q, p ; \tau)$ for the elliptic Apostol sum, although this notation really stands for two different sums. We are sticking to this notation mainly because of the following two reasons: (1) we wish to emphasize the fact that our sum is indeed an elliptic analogue of the classical Apostol sums (which was denoted by $s_{k}(p, q)$ ), and (2) even though there is no single defining formula for elliptic Apostol sums which represent both odd and even parities for $p+q$, we believe there is no danger of confusing the two cases. As we see, the difference is the second factor in each sum, namely, the $(k-1)$-th derivative of the $\varphi$-function in the first case, and the $(k-1)$-th derivative of the $\zeta$-function in the second case.

Remark 2.3. As we will show in Section 6 below, the functions $\varphi(2 \pi i q(\lambda \tau+\mu) / p)$, $\varphi^{(k-1)}(2 \pi i(\lambda \tau+\mu) / p)$ and $\zeta^{(k-1)}(2 \pi i(\lambda \tau+\mu) / p)$ are modular forms for the principal congruence subgroup $\Gamma(p)$ (or $\Gamma(2 p)$ ) of weight $1, k$ and $k$, respectively. In fact, we see that

$$
\zeta^{(k-1)}\left(\frac{2 \pi i(\lambda \tau+\mu)}{p}\right)=-\wp^{(k-2)}\left(\frac{2 \pi i(\lambda \tau+\mu)}{p}\right)
$$

is a "level $p$ Eisenstein series" (cf. [15, pp. 131-135]). Hence $\tilde{s}_{k}(q, p ; \tau)$ is also a modular form for the same congruence subgroup $\Gamma(p)$ (or $\Gamma(2 p)$ ) of weight $k+1$.

Moreover we can show that the limit of $\tilde{s}_{k}(q, p ; \tau)$ is the classical Apostol sum

$$
\lim _{\tau \rightarrow i \infty} \tilde{s}_{2 n-1}(q, p ; \tau)=s_{2 n-1}(q, p)
$$

for any $n>1$. This fact may justify our terminology of "elliptic Apostol sums" for the sums $\tilde{s}_{k}(q, p: \tau)$.

Another fundamental property of Apostol sums is that they satisfy the equality

$$
s_{2 n-1}(q+p, p)=s_{2 n-1}(q, p) .
$$

This implies that an Apostol sum is a (generalized) Dedekind symbol (cf. [10]). As for elliptic Apostol sums, we see that they also satisfy a similar equality:

$$
\tilde{s}_{2 n-1}(q+2 p, p ; \tau)=\tilde{s}_{2 n-1}(q, p ; \tau) .
$$

Indeed, this follows easily from the identity that $\varphi(z+2 \cdot 2 \pi i(m \tau+n))=\varphi(z)$ for $m, n \in \mathbb{Z}$. An implication of this formula is that an elliptic Apostol sum is not a Dedekind symbol.

Recall that if $k$ is even, then the classical Apostol sums are trivial [1, p. 156]. As for the elliptic Apostol sums: if $k$ is even, we have that $\tilde{s}_{k}(q, p ; \tau)=0$. When $k$ is odd, there was a reciprocity law for the Apostol sums. This property should be generalized to the elliptic Apostol sums. Indeed, this is the case, and establishing a reciprocity law for the elliptic Apostol sums is one of our main results and is formulated in the following theorem. 
Theorem 2.2. Let $p$ and $q$ be relatively prime positive integers.

(1) Suppose that $n$ is an integer with $n>0$ and $p+q \equiv 1$ (2). Then we have

$$
\begin{aligned}
& p^{2 n-2} \tilde{s}_{2 n-1}(q, p ; \tau)+q^{2 n-2} \tilde{s}_{2 n-1}(p, q ; \tau) \\
&=\frac{(2 n-1) !}{p q}\left\{\sum_{j=0}^{n} H_{2 j} H_{2 n-2 j} p^{2 j} q^{2 n-2 j}+(2 n-1) H_{2 n}\right\} .
\end{aligned}
$$

(2) Suppose that $n$ is an integer with $n>1$ and $p+q \equiv 0$ (2). Then we have

$$
\begin{aligned}
& p^{2 n-2} \tilde{s}_{2 n-1}(q, p ; \tau)+q^{2 n-2} \tilde{s}_{2 n-1}(p, q ; \tau) \\
&=\frac{(2 n-1) !}{p q}\left\{\sum_{j=0}^{n} H_{2 j} H_{2 n-2 j} p^{2 j} q^{2 n-2 j}-(2 n-1) G_{2 n}\right\} .
\end{aligned}
$$

Remark 2.4. Another interpretation of (2.6) and (2.7) is in terms of modular forms. The left-hand sides of (2.6) and (2.7) represent modular forms for $\Gamma(p)$ if $p$ is even (resp. $\Gamma(2 p)$ if $p$ is odd) and for $\Gamma(q)$ if $q$ is even (resp. $\Gamma(2 q)$ if $q$ is odd) of weight $2 n$, while the right-hand sides represent modular forms for $\Gamma_{0}(2)$ of weight $2 n$. We may view the equations as the identities between these specific modular forms. We are not able to find any literature dealing with these kinds of relations between these specific modular forms, and these relations appear to be new. One intriguing question arises: Is there any geometric interpretation of these formulae?

\section{Proof of Theorem 2.1}

In this section we will give a proof for Theorem 2.1.

Proof of Theorem 2.1(1). We will establish the identity (2.3). We let $f(z)$ and $g(z)$ stand for the left-hand side, and respectively, the right-hand side of (2.3). Namely

$$
\begin{aligned}
f(z)= & \frac{1}{p} \sum_{\substack{\lambda, \mu=0 \\
(\lambda, \mu) \neq(0,0)}}^{p-1}(-1)^{\lambda} \varphi\left(\frac{2 \pi i q(\lambda \tau+\mu)}{p}\right) \varphi\left(z+\frac{2 \pi i(\lambda \tau+\mu)}{p}\right) \\
& +\frac{1}{q} \sum_{\substack{\lambda, \mu=0 \\
(\lambda, \mu) \neq(0,0)}}^{q-1}(-1)^{\lambda} \varphi\left(\frac{2 \pi i p(\lambda \tau+\mu)}{q}\right) \varphi\left(z+\frac{2 \pi i(\lambda \tau+\mu)}{q}\right)
\end{aligned}
$$

and

We claim that

$$
g(z)=-\varphi(p z) \varphi(q z)-\frac{1}{p q} \varphi^{\prime}(z) .
$$

$$
f(z)=g(z) \text {. }
$$

First note that both $f(z)$ and $g(z)$ are meromorphic functions, and both have simple poles at the points $z=-2 \pi i(\lambda \tau+\mu) / p+2 \pi i(m \tau+n)$ with $\lambda, \mu=0,1, \ldots$, $p-1 ;(\lambda, \mu) \neq(0,0) ; m, n \in \mathbb{Z}$ and at $z=-2 \pi i(\lambda \tau+\mu) / q+2 \pi i(m \tau+n)$ with $\lambda, \mu=0,1, \ldots, q-1 ;(\lambda, \mu) \neq(0,0) ; m, n \in \mathbb{Z}$. Furthermore, at these points, both functions have the same residues, that is,

$$
\begin{aligned}
\operatorname{Res}_{z=-2 \pi i(\lambda \tau+\mu) / p+2 \pi i(m \tau+n)}(f) & =\frac{(-1)^{\lambda}}{p} \varphi\left(\frac{2 \pi i q(\lambda \tau+\mu)}{p}\right) \\
& =\operatorname{Res}_{z=-2 \pi i(\lambda \tau+\mu) / p+2 \pi i(m \tau+n)}(g)
\end{aligned}
$$


and

$$
\begin{aligned}
\operatorname{Res}_{z=-2 \pi i(\lambda \tau+\mu) / q+2 \pi i(m \tau+n)}(f) & =\frac{(-1)^{\lambda}}{q} \varphi\left(\frac{2 \pi i p(\lambda \tau+\mu)}{q}\right) \\
& =\operatorname{Res}_{z=-2 \pi i(\lambda \tau+\mu) / q+2 \pi i(m \tau+n)}(g) .
\end{aligned}
$$

Next we investigate other poles. We may assume without loss of generality that $g(z)$ has no pole of order greater than 1. Indeed, the principal parts of $-\varphi(p z) \varphi(q z)$ and $-(1 / p q) \varphi^{\prime}(z)$ at $z=0$ are $-1 /\left(p q z^{2}\right)$ and $1 /\left(p q z^{2}\right)$, respectively, and therefore, they cancel out in $g(z)$. Further notice that the principal part of $-\varphi(p z) \varphi(q z)$ at $z=2 \pi i \tau$ is $1 / p q(z-2 \pi i \tau)^{2}$ (note that $p+q \equiv 1(2)$ ), while the principal part of $-(1 / p q) \varphi^{\prime}(z)$ at $z=2 \pi i \tau$ is $-1 / p q(z-2 \pi i \tau)^{2}$. Hence, again these principal parts at $z=2 \pi i \tau$ cancel out in $g(z)$. Since $g(z)$ is periodic with period $2 \pi i(2 \mathbb{Z} \tau+\mathbb{Z})$, any poles at $z=2 \pi i(m \tau+n)(m, n \in \mathbb{Z})$ also cancel out in $g(z)$. On the other hand, clearly $f(z)$ has no pole of order greater than 1 . Thus it follows that the principal parts of $f(z)$ and $g(z)$ coincide at all of their poles. Therefore $f(z)-g(z)$ is an entire function.

Summarizing the above discussion, we can conclude that $f(z)-g(z)$ is a doubly periodic entire function on $\mathbb{C}$. Then by the well-known Liouville theorem it must be a constant. Hence

$$
f(z)-g(z)=A(p, q ; \tau),
$$

where $A(p, q ; \tau)$ is a constant with respect to $z$.

Next we will determine the constant function $A(p, q ; \tau)$. First note that $\varphi(\tau, z)$ has the expansion at $z=0$ given by the formula (2.2). This implies that the function $g(z)=-\varphi(p z) \varphi(q z)-(1 / p q) \varphi^{\prime}(z)$ has the expansion of the form

$$
g(z)=-\frac{1}{p q z^{2}}\left(1+H_{2} p^{2} z^{2}+\cdots\right)\left(1+H_{2} q^{2} z^{2}+\cdots\right)+\frac{1}{p q z^{2}}\left(1-H_{2} z^{2}-\cdots\right) .
$$

Tending $z \rightarrow 0$, we obtain

$$
\lim _{z \rightarrow 0} g(z)=-\frac{1}{p q}\left(p^{2}+q^{2}\right) H_{2}-\frac{1}{p q} H_{2} .
$$

Now look at the limit of $f(z)$ as $z \rightarrow 0$. We have that $\lim _{z \rightarrow 0} f(z)=f(0)$. Since $A(p, q ; \tau)$ is independent of $z$, we then obtain

$$
\begin{aligned}
A(p, q ; \tau)= & \lim _{z \rightarrow 0}\{f(z)-g(z)\} \\
= & \frac{1}{p} \sum_{\substack{\lambda, \mu=0 \\
(\lambda, \mu) \neq(0,0)}}^{p-1}(-1)^{\lambda} \varphi\left(\frac{2 \pi i q(\lambda \tau+\mu)}{p}\right) \varphi\left(\frac{2 \pi i(\lambda \tau+\mu)}{p}\right) \\
& +\frac{1}{q} \sum_{\substack{\lambda, \mu=0 \\
(\lambda, \mu) \neq(0,0)}}^{q-1}(-1)^{\lambda} \varphi\left(\frac{2 \pi i p(\lambda \tau+\mu)}{q}\right) \varphi\left(\frac{2 \pi i(\lambda \tau+\mu)}{q}\right)+\frac{p^{2}+q^{2}+1}{p q} H_{2} .
\end{aligned}
$$

Proof of Theorem 2.1(1) will be complete if we show that $A(p, q ; \tau)$ is indeed equal to 0 , and this will be done in Lemma 3.1 below. 
Lemma 3.1. Let $p$ and $q$ be relatively prime positive integers such that $p+q \equiv 1(2)$. Then we have

$$
\begin{aligned}
& \frac{1}{p} \sum_{\substack{\lambda, \mu=0 \\
(\lambda, \mu) \neq(0,0)}}^{p-1}(-1)^{\lambda} \varphi\left(\frac{2 \pi i q(\lambda \tau+\mu)}{p}\right) \varphi\left(\frac{2 \pi i(\lambda \tau+\mu)}{p}\right) \\
& \quad+\frac{1}{q} \sum_{\substack{\lambda, \mu=0 \\
(\lambda, \mu) \neq(0,0)}}^{q-1}(-1)^{\lambda} \varphi\left(\frac{2 \pi i p(\lambda \tau+\mu)}{q}\right) \varphi\left(\frac{2 \pi i(\lambda \tau+\mu)}{q}\right)+\frac{p^{2}+q^{2}+1}{p q} H_{2} \\
& \quad=0 .
\end{aligned}
$$

Proof. Let $h(z)$ be an elliptic function defined by

$$
h(z)=\varphi(p z) \varphi(q z) \varphi(z) .
$$

Since $p+q \equiv 1(2), h(z)$ is periodic with period $2 \pi i(\mathbb{Z} \tau+\mathbb{Z})$. Then it follows that the sum of the residues of $h(z)$ in any fundamental domain is zero. For instance, we may take the following fundamental domain:

$$
\left\{2 \pi i(s \tau+t) \mid \frac{1}{2 p q}-1 \leq s, t \leq \frac{1}{2 p q}\right\} .
$$

In this fundamental domain, $h(z)$ has simple poles at $z=-2 \pi i(\lambda \tau+\mu) / p$ with $\lambda, \mu=0,1, \ldots, p-1 ;(\lambda, \mu) \neq(0,0)$ and at $z=-2 \pi i(\lambda \tau+\mu) / q$ with $\lambda, \mu=$ $0,1, \ldots, q-1 ;(\lambda, \mu) \neq(0,0)$. The residues of $h(z)$ at these poles can be computed as follows:

$$
\operatorname{Res}_{z=-2 \pi i(\lambda \tau+\mu) / p}(h)=\frac{(-1)^{\lambda}}{p} \varphi\left(\frac{2 \pi i q(\lambda \tau+\mu)}{p}\right) \varphi\left(\frac{2 \pi i(\lambda \tau+\mu)}{p}\right)
$$

and

$$
\operatorname{Res}_{z=-2 \pi i(\lambda \tau+\mu) / q}(h)=\frac{(-1)^{\lambda}}{q} \varphi\left(\frac{2 \pi i p(\lambda \tau+\mu)}{q}\right) \varphi\left(\frac{2 \pi i(\lambda \tau+\mu)}{q}\right) .
$$

Next we ought to calculate the residue of $h(z)$ at $z=0$. For this we note that $h(z)$ has the following expansion at $z=0$ (which can be derived from the expansion for $\varphi(z)$ given in (2.21) $)$ :

$h(z)=\varphi(p z) \varphi(q z) \varphi(z)=\frac{1}{p q z^{3}}\left(1+H_{2} p^{2} z^{2}+\cdots\right)\left(1+H_{2} q^{2} z^{2}+\cdots\right)\left(1+H_{2} z^{2}+\cdots\right)$.

This enables us to compute the residue of $h(z)$ at $z=0$. We have

$$
\operatorname{Res}_{z=0}(h)=\frac{p^{2}+q^{2}+1}{p q} H_{2} .
$$

Finally summing over the residues for $h(z)$ at all poles, we obtain (3.1) that we are after.

Remark 3.1. The formula in Lemma 3.1 is a special case of Egami's result 8 . Theorem 1]. Unfortunately Egami's original formula as well as its proof contained errors (e.g., the right-hand side of his formula [8, Eq. 5] should be divided by $\left.a_{0} \cdots a_{r}\right)$. In the above lemma and its proof, we reproduced Egami's result by a different method from that of Egami, correcting all the errors in Egami's paper.

We will need another lemma corresponding to Lemma 3.1 to prove the case $p+q \equiv 0(2)$ in Theorem 2.1 2 . 
Lemma 3.2. Let $p$ and $q$ be relatively prime positive integers such that $p+q \equiv 0(2)$. Then we have

(3.2) $\frac{1}{p} \sum_{\substack{\lambda, \mu=0 \\(\lambda, \mu) \neq(0,0)}}^{p-1}(-1)^{\lambda} \varphi\left(\frac{2 \pi i q(\lambda \tau+\mu)}{p}\right)+\frac{1}{q} \sum_{\substack{\lambda, \mu=0 \\(\lambda, \mu) \neq(0,0)}}^{q-1}(-1)^{\lambda} \varphi\left(\frac{2 \pi i p(\lambda \tau+\mu)}{q}\right)=0$.

Proof. Let $h(z)$ be an elliptic function defined by

$$
h(z)=\varphi(p z) \varphi(q z) .
$$

Since $p+q \equiv 0(2), h(z)$ is periodic with period $2 \pi i(\mathbb{Z} \tau+\mathbb{Z})$. Hence the sum of the residues of $h(z)$ in any fundamental domain is zero. We may take the same fundamental domain as in the proof of Lemma 3.1. namely,

$$
\left\{2 \pi i(s \tau+t) \mid \frac{1}{2 p q}-1 \leq s, t \leq \frac{1}{2 p q}\right\} .
$$

In this fundamental domain, $h(z)$ has simple poles at $z=-2 \pi i(\lambda \tau+\mu) / p$ with $\lambda, \mu=0,1, \ldots, p-1 ;(\lambda, \mu) \neq(0,0)$ and at $z=-2 \pi i(\lambda \tau+\mu) / q$ with $\lambda, \mu=$ $0,1, \ldots, q-1 ;(\lambda, \mu) \neq(0,0)$. The residues of $h(z)$ at these poles can be computed as follows:

$$
\operatorname{Res}_{z=-2 \pi i(\lambda \tau+\mu) / p}(h)=\frac{(-1)^{\lambda}}{p} \varphi\left(\frac{2 \pi i q(\lambda \tau+\mu)}{p}\right)
$$

and

$$
\operatorname{Res}_{z=-2 \pi i(\lambda \tau+\mu) / q}(h)=\frac{(-1)^{\lambda}}{q} \varphi\left(\frac{2 \pi i p(\lambda \tau+\mu)}{q}\right) .
$$

We need to calculate the residue of $h(z)$ at $z=0$. For this we expand $h(z)$ at $z=0$ :

$$
h(z)=\varphi(p z) \varphi(q z)=\frac{1}{p q z^{2}}\left(1+H_{2} p^{2} z^{2}+\cdots\right)\left(1+H_{2} q^{2} z^{2}+\cdots\right) .
$$

Then we obtain that $\operatorname{Res}_{z=0}(h)=0$. Finally summing over the residues for $h(z)$ at all poles, we arrive at the equation (3.2).

In the rest of this section, we will give a proof for Theorem 2.1 (2).

Proof of Theorem 2.1 (2). We will establish the identity (2.4) in the case $p+q \equiv$ 0 (2). Our proof is along the same line as for Theorem 2.1(1) in the case $p+q \equiv 1$ (2) (although we need to use the first derivatives $f^{\prime}(z)$ and $g^{\prime}(z)$ in place of $f(z)$ and $g(z)$ to establish the assertion that $f(z)-g(z)$ is a constant). Let $f(z)$ and $g(z)$ be the functions defined, respectively, as follows:

$$
\begin{aligned}
f(z)= & \frac{1}{p} \sum_{\substack{\lambda, \mu=0 \\
(\lambda, \mu) \neq(0,0)}}^{p-1}(-1)^{\lambda} \varphi\left(\frac{2 \pi i q(\lambda \tau+\mu)}{p}\right) \zeta\left(z+\frac{2 \pi i(\lambda \tau+\mu)}{p}\right) \\
& +\frac{1}{q} \sum_{\substack{\lambda, \mu=0 \\
(\lambda, \mu) \neq(0,0)}}^{q-1}(-1)^{\lambda} \varphi\left(\frac{2 \pi i p(\lambda \tau+\mu)}{q}\right) \zeta\left(z+\frac{2 \pi i(\lambda \tau+\mu)}{q}\right)
\end{aligned}
$$

and

$$
g(z)=-\varphi(p z) \varphi(q z)-\frac{1}{p q} \zeta^{\prime}(z)
$$


We claim that

$$
f(z)-g(z) \text { is a constant. }
$$

First note that both $f(z)$ and $g(z)$ are meromorphic functions, and both have simple poles at the points $z=-2 \pi i(\lambda \tau+\mu) / p+2 \pi i(m \tau+n)$ with $\lambda, \mu=0,1, \ldots$, $p-1 ;(\lambda, \mu) \neq(0,0) ; m, n \in \mathbb{Z}$ and at $z=-2 \pi i(\lambda \tau+\mu) / q+2 \pi i(m \tau+n)$ with $\lambda, \mu=0,1, \ldots, q-1 ;(\lambda, \mu) \neq(0,0) ; m, n \in \mathbb{Z}$. Furthermore, the residues at these points are equal, that is,

$$
\begin{aligned}
\operatorname{Res}_{z=-2 \pi i(\lambda \tau+\mu) / p+2 \pi i(m \tau+n)}(f) & =\frac{(-1)^{\lambda}}{p} \varphi\left(\frac{2 \pi i q(\lambda \tau+\mu)}{p}\right) \\
& =\operatorname{Res}_{z=-2 \pi i(\lambda \tau+\mu) / p+2 \pi i(m \tau+n)}(g)
\end{aligned}
$$

and

$$
\begin{aligned}
\operatorname{Res}_{z=-2 \pi i(\lambda \tau+\mu) / q+2 \pi i(m \tau+n)}(f) & =\frac{(-1)^{\lambda}}{q} \varphi\left(\frac{2 \pi i p(\lambda \tau+\mu)}{q}\right) \\
& =\operatorname{Res}_{z=-2 \pi i(\lambda \tau+\mu) / q+2 \pi i(m \tau+n)}(g) .
\end{aligned}
$$

Next we investigate other poles. We may assume without loss of generality that $g(z)$ has no pole of order greater than 1. Indeed, the principal parts of $-\varphi(p z) \varphi(q z)$ and $-(1 / p q) \zeta^{\prime}(z)$ at $z=2 \pi i(m \tau+n)(m, n \in \mathbb{Z})$ are $-1 / p q(z-(m \tau+n))^{2}$ and $1 / p q(z-(m \tau+n))^{2}$, respectively, and moreover, they cancel out in $g(z)$. (This can be seen easily at $z=0$. At $z \neq 0$, this follows from noticing that $g(z)$ is periodic with period $2 \pi i(\mathbb{Z} \tau+\mathbb{Z})$ (because of the hypothesis $p+q \equiv 0(2)$ ).) Clearly $f(z)$ has no pole of order greater than 1. Hence the principal parts of $f(z)$ and $g(z)$ coincide at all of their poles. Therefore $f(z)-g(z)$ is an entire function.

Now note that $\zeta(z)$ is not periodic, but if we take the derivative $\zeta^{\prime}(z)$, then it is periodic with period $2 \pi i(\mathbb{Z} \tau+\mathbb{Z})$. Take the first derivative $f^{\prime}(z)-g^{\prime}(z)$ of $f(z)-g(z)$. Then $f^{\prime}(z)-g^{\prime}(z)$ is periodic with period $2 \pi i(\mathbb{Z} \tau+\mathbb{Z})$. Thus we can conclude that $f^{\prime}(z)-g^{\prime}(z)$ is a doubly periodic entire function on $\mathbb{C}$, and then it must be a constant by the well-known Liouville Theorem. Hence we may write

$$
f(z)-g(z)=B(p, q ; \tau) z+C(p, q ; \tau),
$$

where $B(p, q ; \tau)$ and $C(p, q ; \tau)$ are constants with respect to $z$.

We claim that $B(p, q ; \tau)=0$.

By the periodicity of $\varphi$ and the quasi-periodicity of $\zeta$, we have:

$$
\zeta(z+2 \pi i)=\zeta(z)+\eta_{1}, \varphi(z+2 \pi i)=\varphi(z) \text { and } \zeta^{\prime}(z+2 \pi i)=\zeta^{\prime}(z) .
$$

This gives rise to the identity

$$
\begin{aligned}
& 2 \pi i B(p, q ; \tau)=\{f(z+2 \pi i)-g(z+2 \pi i)\}-\{f(z)-g(z)\} \\
&=\eta_{1}\left\{\frac{1}{p} \sum_{\substack{\lambda, \mu=0 \\
(\lambda, \mu) \neq(0,0)}}^{p-1}(-1)^{\lambda} \varphi\left(\frac{2 \pi i q(\lambda \tau+\mu)}{p}\right)\right. \\
&\left.+\frac{1}{q} \sum_{\substack{\lambda, \mu=0 \\
(\lambda, \mu) \neq(0,0)}}^{q-1}(-1)^{\lambda} \varphi\left(\frac{2 \pi i p(\lambda \tau+\mu)}{q}\right)\right\} \\
&=0 .
\end{aligned}
$$


The second identity is derived from (3.3) and the third from Lemma [3.2. This proves our claim that $B(p, q ; \tau)=0$.

Finally this establish (3.2) that we are after.

Remark 3.2. At the moment we do not know how to determine the exact value of the constant $C(p, q ; \tau)$. The difficulty stems from the fact that $\zeta(z)$ is only quasi-periodic and that we do not have a reciprocity law for

$$
\frac{1}{p} \sum_{\substack{\lambda, \mu=0 \\(\lambda, \mu) \neq(0,0)}}^{p-1}(-1)^{\lambda} \varphi\left(\frac{2 \pi i q(\lambda \tau+\mu)}{p}\right) \zeta\left(\frac{2 \pi i(\lambda \tau+\mu)}{p}\right) .
$$

\section{Proof of Theorem 2.2}

In this section we will give a proof for Theorem 2.2.

Let $f(z)$ be a meromorphic function on $\mathbb{C}$. (In what follows we will consider the case where $f(z)=\varphi(z)$ or $\zeta(z)$.) If $f(z)$ does not have a pole at $z=\alpha$, then $f(z)$ can be expanded at $z=\alpha$ :

$$
f(z+\alpha)=f(\alpha)+\sum_{k=1}^{\infty} \frac{f^{(k)}(\alpha)}{k !} z^{k} .
$$

We now give proof for Theorem 2.2(1), that is, the case $p+q \equiv 1(2)$.

Proof of Theorem 2.2(1). We expand both sides of (2.3) and compare the coefficients of $z^{2 n-2}$ to obtain the claimed identity (2.66).

The first term of the left-hand side of (2.3) is expanded as follows:

$$
\begin{aligned}
& \frac{1}{p} \sum_{\substack{\lambda, \mu=0 \\
(\lambda, \mu) \neq(0,0)}}^{p-1}(-1)^{\lambda} \varphi\left(\frac{2 \pi i q(\lambda \tau+\mu)}{p}\right) \varphi\left(z+\frac{2 \pi i(\lambda \tau+\mu)}{p}\right) \\
& =\frac{1}{p} \sum_{\substack{\lambda, \mu=0 \\
(\lambda, \mu) \neq(0,0)}}^{p-1}(-1)^{\lambda} \varphi\left(\frac{2 \pi i q(\lambda \tau+\mu)}{p}\right) \varphi\left(\frac{2 \pi i(\lambda \tau+\mu)}{p}\right) \\
& +\sum_{k=1}^{\infty} \frac{1}{k !}\left\{\frac{1}{p} \sum_{\substack{\lambda, \mu=0 \\
(\lambda, \mu) \neq(0,0)}}^{p-1}(-1)^{\lambda} \varphi\left(\frac{2 \pi i q(\lambda \tau+\mu)}{p}\right) \varphi^{(k)}\left(\frac{2 \pi i(\lambda \tau+\mu)}{p}\right)\right\} z^{k} .
\end{aligned}
$$

We obtain a similar expansion for the second term of the left-hand side of (2.3). Then the coefficient of $z^{2 n-2}$ in the left-hand side of (2.3) is given by

$$
\left.\begin{array}{r}
\frac{1}{(2 n-2) !}\left\{\frac{1}{p} \sum_{\substack{\lambda, \mu=0 \\
(\lambda, \mu) \neq(0,0)}}^{p-1}(-1)^{\lambda} \varphi\left(\frac{2 \pi i q(\lambda \tau+\mu)}{p}\right) \varphi^{(2 n-2)}\left(\frac{2 \pi i(\lambda \tau+\mu)}{p}\right)\right. \\
+\frac{1}{q} \sum_{\substack{\lambda, \mu=0 \\
(\lambda, \mu) \neq(0,0)}}^{q-1}(-1)^{\lambda} \varphi\left(\frac{2 \pi i p(\lambda \tau+\mu)}{q}\right) \varphi^{(2 n-2)}\left(\frac{2 \pi i(\lambda \tau+\mu)}{q}\right)
\end{array}\right\} .
$$


Further we see by Definition 2.1 that this is equal to

$$
-\frac{1}{(2 n-1) !}\left\{p^{2 n-2} \tilde{s}_{2 n-1}(q, p ; \tau)+q^{2 n-2} \tilde{s}_{2 n-1}(p, q ; \tau)\right\} .
$$

Next we expand the right-hand side of (2.3) into the following form:

$$
\begin{aligned}
-\varphi(p z) \varphi(q z)-\frac{1}{p q} \varphi^{\prime}(z) & \\
=- & \frac{1}{p q z^{2}}\left(1+H_{2} p^{2} z^{2}+H_{4} p^{4} z^{4}+\cdots\right)\left(1+H_{2} q^{2} z^{2}+H_{4} q^{4} z^{4}+\cdots\right) \\
& \quad+\frac{1}{p q z^{2}}\left(1-H_{2} z^{2}-3 H_{4} z^{4}-5 H_{6} z^{6}-\cdots\right) .
\end{aligned}
$$

Then the coefficient of $z^{2 n-2}$ in the right-hand side of (2.3) is given by

$$
-\frac{1}{p q} \sum_{j=0}^{n} H_{2 j} H_{2 n-2 j} p^{2 j} q^{2 n-2 j}-\frac{1}{p q}(2 n-1) H_{2 n} .
$$

Now comparing the coefficients of $z^{2 n-2}$ in both sides of (2.3), we see that the expressions (4.2) and (4.3) are indeed equal. This yields the identity of Theorem $2.2(1)$.

Proof of Theorem 2.2(2). The proof is similar to that of Theorem2.2(1). The detail is left to the reader.

\section{Limits OF ELLIPTIC RECIPROCITY LAWS}

In this section we will calculate the limits of elliptic reciprocity laws (2.3) and (2.4) as $\tau \rightarrow i \infty$. We show that the limits indeed coincide with the original reciprocity law for Apostol sums (1.2). This fact may justify our terminology that Definition 2.1 is an elliptic analogue of Apostol sums.

First we recall a number of formulae for elliptic functions. Put

$$
q=e^{2 \pi i \tau} \text { and } \xi=e^{z} .
$$

Then the functions $\varphi(z)$ and $\zeta(z)$ have the following expansion (see [13, pp.144,147]):

$$
\begin{gathered}
\varphi(z)=\frac{1}{2} \frac{\xi^{1 / 2}+\xi^{-1 / 2}}{\xi^{1 / 2}-\xi^{-1 / 2}} \prod_{n=1}^{\infty} \frac{\left(1+q^{n} \xi\right)\left(1+q^{n} \xi^{-1}\right)\left(1-q^{n}\right)^{2}}{\left(1-q^{n} \xi\right)\left(1-q^{n} \xi^{-1}\right)\left(1+q^{n}\right)^{2}} \\
\zeta(z)=2 G_{2} z+\frac{1}{2} \frac{\xi^{1 / 2}+\xi^{-1 / 2}}{\xi^{1 / 2}-\xi^{-1 / 2}}+\sum_{n=1}^{\infty}\left(\frac{-q^{n} \xi}{1-q^{n} \xi}+\frac{q^{n} \xi^{-1}}{1-q^{n} \xi^{-1}}\right) .
\end{gathered}
$$

Define yet another function $\omega(z)$ by

$$
\omega(z):=\zeta(z)-2 G_{2} z\left(=\frac{1}{2} \frac{\xi^{1 / 2}+\xi^{-1 / 2}}{\xi^{1 / 2}-\xi^{-1 / 2}}+\sum_{n=1}^{\infty}\left(\frac{-q^{n} \xi}{1-q^{n} \xi}+\frac{q^{n} \xi^{-1}}{1-q^{n} \xi^{-1}}\right)\right) .
$$

We are interested in the limits of $\varphi(z)$ and $\omega(z)$ for $z=2 \pi i(x \tau+y)$ as $\tau \rightarrow i \infty$.

Lemma 5.1.

(1) Suppose that $x \geq 0$. Then

$$
\lim _{\tau \rightarrow i \infty} \varphi(2 \pi i(x \tau+y))= \begin{cases}-\frac{1}{2}(-1)^{[x]}, & x \notin \mathbb{Z}, \\ \frac{1}{2 i} \cot (\pi y), & x \in \mathbb{Z} .\end{cases}
$$

Here $[x]$ denotes the largest integer not exceeding $x$. 
(2) Suppose that $0 \leq x<1$. Then

$$
\lim _{\tau \rightarrow i \infty} \omega(2 \pi i(x \tau+y))= \begin{cases}-\frac{1}{2}, & 0<x<1, \\ \frac{1}{2 i} \cot (\pi y), & x=0 .\end{cases}
$$

Proof. (1) This limit is obtained by Egami in [8, p. 102], using the expansion (5.2).

(2) This limit can be proved similarly as for (1). In fact, the terms

$$
\sum_{n=1}^{\infty}\left(\frac{-q^{n} \xi}{1-q^{n} \xi}+\frac{q^{n} \xi^{-1}}{1-q^{n} \xi^{-1}}\right)
$$

in (5.3) tend to 0 as $\tau \rightarrow i \infty$. Furthermore, for $z=2 \pi i(x \tau+y)$, we see that as $\tau \rightarrow i \infty$,

$$
\frac{1}{2} \frac{\xi^{1 / 2}+\xi^{-1 / 2}}{\xi^{1 / 2}-\xi^{-1 / 2}}=\frac{1}{2} \frac{e^{2 \pi i(x \tau+y)}+1}{e^{2 \pi i(x \tau+y)}-1} \rightarrow \begin{cases}-\frac{1}{2}, & 0<x<1, \\ \frac{1}{2} \frac{e^{2 \pi i y}+1}{e^{2 \pi i y}-1}=\frac{1}{2 i} \cot (\pi y), & x=0 .\end{cases}
$$

These facts yield the limit as claimed.

Now we apply Lemma 5.1 to obtain the limits of elliptic reciprocity laws (2.3) and (2.4) as $\tau \rightarrow i \infty$.

The case $p+q \equiv 1(2)$. Consider the case $p+q \equiv 1(2)$. We see that the left-hand side of the identity (2.3) has the following limit when $\tau \rightarrow i \infty$ :

$$
\begin{aligned}
\frac{1}{p} \sum_{\substack{\lambda, \mu=0 \\
(\lambda, \mu) \neq(0,0)}}^{p-1}(-1)^{\lambda} \varphi\left(\frac{2 \pi i q(\lambda \tau+\mu)}{p}\right) \varphi\left(z+\frac{2 \pi i(\lambda \tau+\mu)}{p}\right) \\
=\frac{1}{p} \sum_{\mu=0}^{p-1} \sum_{\lambda=1}^{p-1}(-1)^{\lambda} \varphi\left(\frac{2 \pi i q(\lambda \tau+\mu)}{p}\right) \varphi\left(z+\frac{2 \pi i(\lambda \tau+\mu)}{p}\right) \\
\quad+\frac{1}{p} \sum_{\mu=1}^{p-1} \varphi\left(\frac{2 \pi i q \mu}{p}\right) \varphi\left(z+\frac{2 \pi i \mu}{p}\right) \\
\rightarrow-\frac{1}{4} \sum_{\lambda=1}^{p-1}(-1)^{\lambda+1+[q \lambda / p]}+\frac{1}{p} \sum_{\mu=1}^{p-1} \frac{1}{2 i} \cot \left(\frac{\pi q \mu}{p}\right) \frac{1}{2 i} \cot \left(\frac{z}{2 i}+\frac{\pi \mu}{p}\right)
\end{aligned}
$$

On the other hand, noting that $\cot ^{\prime}(z)=-\csc ^{2}(z)$, we see that the right-hand side of the identity (2.3) tends to the following limit when $\tau \rightarrow i \infty$ :

$$
-\varphi(p z) \varphi(q z)-\frac{1}{p q} \varphi^{\prime}(z) \rightarrow-\frac{1}{2 i} \cot \left(\frac{p z}{2 i}\right) \frac{1}{2 i} \cot \left(\frac{q z}{2 i}\right)+\frac{1}{p q} \frac{1}{(2 i)^{2}} \csc ^{2}\left(\frac{z}{2 i}\right) .
$$

We also need to recall the fact due to Berndt-Evans [5, p. 3] that for relatively prime integers $p$ and $q$ with $p+q \equiv 1(2)$, the sum $\sum_{\lambda=1}^{p-1}(-1)^{\lambda+1+[q \lambda / p]}$ in (5.4) satisfies the following reciprocity law:

$$
\sum_{\lambda=1}^{p-1}(-1)^{\lambda+1+[q \lambda / p]}+\sum_{\lambda=1}^{q-1}(-1)^{\lambda+1+[p \lambda / q]}=1 .
$$

Then concocting (5.4), (5.5) and (5.6), and rescaling the variable $z$ by $z / 2 i$, we arrive at the reciprocity law given in (1.2). 
Furthermore, expanding the last term in (5.4) and then showing that its coefficient of $z^{2 n-2}$ is equal to $\left(-p^{2 n-2} /(2 n-1) !\right) s_{2 n-1}(q, p)$ (cf. [12] 11, 2] for detailed calculations), we obtain (2.5):

$$
\lim _{\tau \rightarrow i \infty} \tilde{s}_{2 n-1}(q, p ; \tau)=s_{2 n-1}(q, p) .
$$

This proves our assertion that Theorem 2.1(1) is an elliptic analogue of the classical reciprocity law (1.2) in the case $p+q \equiv 1(2)$.

The case $p+q \equiv 0(2)$. Now consider the case that $p+q \equiv 0(2)$. First note that we can reformulate (2.4) in the following form:

$$
\begin{gathered}
\frac{1}{p} \sum_{\substack{\lambda, \mu=0 \\
(\lambda, \mu) \neq(0,0)}}^{p-1}(-1)^{\lambda} \varphi\left(\frac{2 \pi i q(\lambda \tau+\mu)}{p}\right)\left\{\zeta\left(z+\frac{2 \pi i(\lambda \tau+\mu)}{p}\right)-2 G_{2}\left(z+\frac{2 \pi i(\lambda \tau+\mu)}{p}\right)\right\} \\
+\frac{1}{q} \sum_{\substack{\lambda, \mu=0 \\
(\lambda, \mu) \neq(0,0)}}^{q-1}(-1)^{\lambda} \varphi\left(\frac{2 \pi i p(\lambda \tau+\mu)}{q}\right)\left\{\zeta\left(z+\frac{2 \pi i(\lambda \tau+\mu)}{q}\right)-2 G_{2}\left(z+\frac{2 \pi i(\lambda \tau+\mu)}{q}\right)\right\} \\
=-\varphi(p z) \varphi(q z)-\frac{1}{p q} \zeta^{\prime}(z)+D(p, q ; \tau) .
\end{gathered}
$$

To obtain this reformulation, use the following identity (which is deduced from (3.2)):

$$
\left\{\frac{1}{p} \sum_{\substack{\lambda, \mu=0 \\(\lambda, \mu) \neq(0,0)}}^{p-1}(-1)^{\lambda} \varphi\left(\frac{2 \pi i q(\lambda \tau+\mu)}{p}\right)+\frac{1}{q} \sum_{\substack{\lambda, \mu=0 \\(\lambda, \mu) \neq(0,0)}}^{q-1}(-1)^{\lambda} \varphi\left(\frac{2 \pi i p(\lambda \tau+\mu)}{q}\right)\right\} G_{2}
$$

Here $D(p, q ; \tau)$ is a constant with respect to $z$. Actually, $D(p, q ; \tau)$ is explicitly given as follows:

$$
\begin{aligned}
D(p, q ; \tau)=C(p, q ; \tau) & -2\left\{\frac{1}{p} \sum_{\substack{\lambda, \mu=0 \\
(\lambda, \mu) \neq(0,0)}}^{p-1}(-1)^{\lambda} \varphi\left(\frac{2 \pi i q(\lambda \tau+\mu)}{p}\right) \frac{2 \pi i(\lambda \tau+\mu)}{p}\right. \\
& \left.+\frac{1}{q} \sum_{\substack{\lambda, \mu=0 \\
\lambda, \mu) \neq(0,0)}}^{q-1}(-1)^{\lambda} \varphi\left(\frac{2 \pi i p(\lambda \tau+\mu)}{q}\right) \frac{2 \pi i(\lambda \tau+\mu)}{q}\right\} G_{2} .
\end{aligned}
$$

Now we are ready to prove that (5.7) is an elliptic analogue of the classical reciprocity law (1.2) (up to addition of constant) in the case $p+q \equiv 0$ (2). We are interested in evaluating the limits of both sides of (5.7) as $\tau \rightarrow i \infty$. For this first note that $\zeta(z)-2 G_{2} z=\omega(z)$. Now we apply Lemma 5.1(2) to (5.7). The left-hand 
side of (5.7) tends to the following limit when $\tau \rightarrow i \infty$ :

$$
\begin{aligned}
-\frac{1}{4} \sum_{\lambda=1}^{p-1}(-1)^{\lambda+1+[q \lambda / p]}+\frac{1}{p} \sum_{\mu=1}^{p-1} \frac{1}{2 i} \cot \left(\frac{\pi q \mu}{p}\right) \frac{1}{2 i} \cot \left(\frac{z}{2 i}+\frac{\pi \mu}{p}\right) \\
-\frac{1}{4} \sum_{\lambda=1}^{q-1}(-1)^{\lambda+1+[p \lambda / q]}+\frac{1}{q} \sum_{\mu=1}^{q-1} \frac{1}{2 i} \cot \left(\frac{\pi p \mu}{q}\right) \frac{1}{2 i} \cot \left(\frac{z}{2 i}+\frac{\pi \mu}{q}\right) .
\end{aligned}
$$

On the other hand, to find the limit for the right-hand side of (5.7) as $\tau \rightarrow i \infty$, we will make use of the fact that

$$
\lim _{\tau \rightarrow i \infty}-\zeta^{\prime}(z)=\lim _{\tau \rightarrow i \infty} \wp(z)=\frac{1}{(2 i)^{2}} \csc ^{2}\left(\frac{z}{2 i}\right) .
$$

Then the right-hand side of (5.7) approaches the limit

$$
-\frac{1}{2 i} \cot \left(\frac{p z}{2 i}\right) \frac{1}{2 i} \cot \left(\frac{q z}{2 i}\right)+\frac{1}{p q} \frac{1}{(2 i)^{2}} \csc ^{2}\left(\frac{z}{2 i}\right)+c(p, q),
$$

where $c(p, q)$ is a constant with respect to $z$.

Finally concocting (5.8), (5.9) and (5.6), and rescaling the variable $z$ by $z / 2 i$, we arrive at the reciprocity law (1.2) in the case $p+q \equiv 0(2)$.

We summarize the above discussion in the following proposition.

Proposition 5.2. (a) Suppose that $p+q \equiv 1(2)$. Then the limit of (2.3) as $\tau \rightarrow i \infty$ yields the identity (1.2) on cotangent sums.

(b) Suppose that $p+q \equiv 0(2)$. Then the limit of (5.7) (equivalently (2.4)) as $\tau \rightarrow i \infty$ yields the identity (1.2) on cotangent sums.

Therefore, (2.3) and (2.4) are indeed elliptic analogues of the classical reciprocity laws for Apostol sums.

\section{NeW RELATions BeTWEen MOdUlar FORMS}

We can state some new relations between modular forms from the reciprocity laws established in Theorem 2.2. Here we give some interpretation of our results from the point of view of modular forms. Let $\mathbb{H}$ be the upper half complex plane. A function $f: \mathbb{H} \times \mathbb{C} \rightarrow \mathbb{C}$ is called a Jacobi form of weight $k$ and index 0 for the congruence subgroup $\Gamma$ of $S L_{2}(\mathbb{Z})$ if it satisfies the following two conditions:

$$
\begin{gathered}
f\left(\frac{a \tau+b}{c \tau+d}, \frac{z}{c \tau+d}\right)=(c \tau+d)^{k} f(\tau, z) \quad\left(\left(\begin{array}{ll}
a & b \\
c & d
\end{array}\right) \in \Gamma\right), \\
f(\tau, z+2 \pi i(m \tau+n))=f(\tau, z) \quad(m, n \in \mathbb{Z})
\end{gathered}
$$

together with some regularity conditions. The reader is referred to $[9,13$ for more detailed accounts about Jacobi forms.

It is known that the function $\varphi$ is a Jacobi form of weight 1 and index 0 for $\Gamma_{0}(2)$ while the Weierstrass $\wp$-function is a Jacobi form of weight 2 and index 0 for $S L_{2}(\mathbb{Z})$ (see $[13$ and $[9]$ ). It is easily shown that if $f$ is a Jacobi form of weight $w$ and index 0 , then $f^{(k)}$ is a Jacobi form of weight $w+k$ and index 0 . Hence $\varphi^{(k)}$ and $\zeta^{(k)}=-\wp^{(k-1)}$ are Jacobi forms of weight $k+1$ and index 0 . 
Lemma 6.1. Suppose that $f(\tau, z)$ is a Jacobi form of weight $k$ and index 0 for $S L_{2}(\mathbb{Z})$, and suppose that it has poles only at $z=2 \pi i(\mathbb{Z} \tau+\mathbb{Z})$. Then, for $\lambda, \mu=$ $0,1, \ldots, p-1 ;(\lambda, \mu) \neq(0,0), f(\tau, 2 \pi i(\lambda \tau+\mu) / p)$ is a modular form for $\Gamma(p)$ of weight $k$.

Proof. This follows from the following identities for $\left(\begin{array}{ll}a & b \\ c & d\end{array}\right) \in \Gamma(p)$ :

$$
\begin{aligned}
f\left(\frac{a \tau+b}{c \tau+d}, \frac{2 \pi i\left(\lambda \frac{a \tau+b}{c \tau+d}+\mu\right)}{p}\right) & =f\left(\frac{a \tau+b}{c \tau+d}, \frac{2 \pi i}{c \tau+d} \frac{\lambda(a \tau+b)+\mu(c \tau+d)}{p}\right) \\
& =(c \tau+d)^{k} f\left(\tau, \frac{2 \pi i\{\lambda(a \tau+b)+\mu(c \tau+d)\}}{p}\right) \\
& =(c \tau+d)^{k} f\left(\tau, \frac{2 \pi i(\lambda \tau+\mu)}{p}\right) .
\end{aligned}
$$

(Notice that the second identity follows from (6.1), and the third from (6.2).)

Similarly we know that if $f(\tau, z)$ is a Jacobi form of weight $k$ and index 0 for $\Gamma_{0}(2), f(\tau, 2 \pi i(\lambda \tau+\mu) / p)$ is a modular form of weight $k$ for $\Gamma(p)($ resp. $\Gamma(2 p))$ if $p$ is even (resp. odd).

Now we apply the above argument to $f=\varphi$, or $\varphi^{(k)}$ or $\zeta^{(k)}$.

Proposition 6.2. The elliptic Apostol sum $\tilde{s}_{2 n-1}(q, p ; \tau)$ is a modular form of weight $2 n$ for $\Gamma(p)$ (resp. $\Gamma(2 p)$ ) if $p$ is even (resp. odd).

Since $H_{2 n}$ is a modular form for $\Gamma_{0}(2)$, the reciprocity law for elliptic Apostol sums (Theorem 2.2) gives rise to new relations between modular forms for $\Gamma(p)$ (or $\Gamma(2 p)$ if $p$ is odd), $\Gamma(q)$ (or $\Gamma(2 q)$ if $q$ is odd) and $\Gamma_{0}(2)$.

Remark 6.1. The classical Dedekind sums $s(q, p)$ first appeared in the transformation formula of $\log \eta(\tau)$. The Apostol sums $s_{k}(q, p)$ also appeared in the transformation formula of some kinds of Lambert series. We will end the paper by posing a natural question.

Question. The classical and generalized Dedekind sums appeared in a formula due to Mordell [17], which counts the number of lattice points in a tetrahedron. Also there are many generalizations of Mordell's formula to higher-dimensional cases. Is there any geometrical interpretation of the elliptic Apostol sums introduced in this paper?

Another question is: Are there generalizations of Lambert series whose transformation rules are described in terms of elliptic Apostol sums?

\section{ACKNOWLEDGMENTS}

We are grateful to the referee for bringing the paper of Bayad [3] to our attention, and for his further comments and valuable suggestions, which improved and clarified our presentation.

\section{REFERENCES}

[1] Apostol, T. M.: Generalized Dedekind sums and transformation formulae of certain Lambert series. Duke Math. J. 17, 147-157 (1950). MR 11:641g

[2] Apostol, T. M.: Theorems on generalized Dedekind sums. Pacific J. Math. 2, 1-9 (1952). MR 13:725c

[3] Bayad, A.: Sommes de Dedekind elliptiques et formes de Jacobi. Ann. Inst. Fourier, Grenoble 51, 29-42 (2001). MR 2002d:11049 
[4] Berndt, B. C.: Reciprocity theorems for Dedekind sums and generalizations. Advances in Math. 23, 285-316 (1977). MR 55:2722

[5] Berndt, B. C., Evans, R. J.: On Rademacher's multiplier system for the classical thetafunction. Contemp. Math. 166, 1-7 (1994) MR 96b:11060

[6] Carlitz, L.: On reciprocity theorem for Dedekind sums. Pacific J. Math. 3, 523-527 (1952) MR 15:12c

[7] Dieter, U.: Cotangent sums, a further generalization of Dedekind sums. J. Number Theory 18, 289-305 (1984). MR 85i:11042

[8] Egami, S.: An elliptic analogue of the multiple Dedekind sums. Compos. Math. 99, 99-103 (1995). MR 96g:11040

[9] Eichler, M., Zagier, D.: The Theory of Jacobi Forms. Boston-Basel-Stuttgart: Birkhäuser 1985. MR 86j:11043

[10] Fukuhara, S.: Modular forms, generalized Dedekind symbols and period polynomials. Math. Ann. 310, 83-101 (1998). MR 99k:11061

[11] Fukuhara, S.: Dedekind symbols associated with J-forms and their reciprocity law. J. Number Theory 98, 236-253 (2003).

[12] Fukuhara, S.: New trigonometric identities and generalized Dedekind sums. Tokyo J. Math. 26, 1-14 (2003).

[13] Hirzebruch, F., Berger, T., Jung, R.: Manifolds and Modular Forms. Bonn: Vieweg 1992. MR 94d:57001

[14] Ito, H.: On a property of elliptic Dedekind sums. J. Number Theory 27, 17-21 (1987). MR 89a: 11050

[15] Koblitz, N.: Introduction to Elliptic Curves and Modular Forms (Graduate Texts in Math. 97). Springer-Verlag 1993. MR 94a:11078

[16] Mikolás, M.: On certain sums generating the Dedekind sums and their reciprocity laws. Pacific J. Math. 7, 1167-1178 (1957). MR 19:943c

[17] Mordell, L. J.: Lattice points in a tetrahedron and generalized Dedekind sums. J. London Math. Soc. (N.S.) 15, 41-46 (1951). MR 13:322b

[18] Rademacher, H., Grosswald, E.: Dedekind Sums (Carus Math. Mono. No. 16). Math. Assoc. Amer. 1972. MR 50:9767

[19] Sczech, R.: Dedekindsummen mit elliptischen Funktionen. Invent. Math. 67, 523-551 (1984). MR 86h:11037

[20] Zagier, D.: Higher dimensional Dedekind sums. Math. Ann. 202, 149-172 (1973). MR 50:9801

Department of Mathematics, Tsuda College, Tsuda-machi 2-1-1, Kodaira-Shi, Tokyo 187-8577, JAPAN

E-mail address: fukuhara@tsuda.ac.jp

Department of Mathematics and Statistics, Queen's University, Kingston, Ontario, Canada K7L 3N6

E-mail address: yui@mast.queensu.ca 